J. Astrophys. Astr. (0000) 00, 000-000

\title{
High Resolution Observations using Adaptive Optics: Achievements and Future Needs
}

\author{
K. Sankarasubramanian ${ }^{1} \&$ T. Rimmele ${ }^{2}$ \\ ${ }^{1}$ ISRO Satellite Centre, Bangalore 560 017, India, email: sankark@isac.gov.in \\ ${ }^{2}$ National Solar Observatory*, Sunspot, NM - 88349, USA
}

2018 October 26

\begin{abstract}
Over the last few years, several interesting observations were obtained with the help of solar Adaptive Optics (AO). In this paper, few observations made using the solar $\mathrm{AO}$ are enlightened and briefly discussed. A list of disadvantages with the current $\mathrm{AO}$ system are presented. With telescopes larger than $1.5 \mathrm{~m}$ are expected during the next decade, there is a need to develop the existing AO technologies for large aperture telescopes. Some aspects of this development are highlighted. Finally, the recent AO developments in India are also presented.

Key words: Small-scale structures - Adaptive Optics - MultiConjugate Adaptive Optics - High Spatial Resolution
\end{abstract}

\section{Introduction}

Sun provides an unique opportunity for greater understanding of physical processes happening in stellar atmospheres. The solar atmosphere is structured to very small scales which are dynamic in nature. Understanding the nature of these small-scale structures and dynamics may provide important clues to some puzzling and unanswered questions. It is now very well realised that the small-scale dynamics can influence the large-scale structures (DeRosa, 2005). In hydrodynamic condition, two important scales determine the structuring of the solar atmosphere: (i) Pressure scale height, and (ii) Photon mean free path. Both these scales correspond to a value of $70 \mathrm{~km}$ (i.e., about 0."1) at the photosphere. However, smaller magnetic structures are seen in realistic numerical magneto-hydrodynamic simulations (Stein \& Nordlund, 2006; Schussler \& Vogler, 2006). Grid sizes used in these simulations are smaller than $0 . " 1$ and future simulations are aimed to achieve a grid size of $0 . " 02$ or better.

${ }^{*} \mathrm{NSO}$ is operated by the Association of Universities for Research in Astronomy (AURA) under cooperative agreement with National Science Foundation (NSF) 

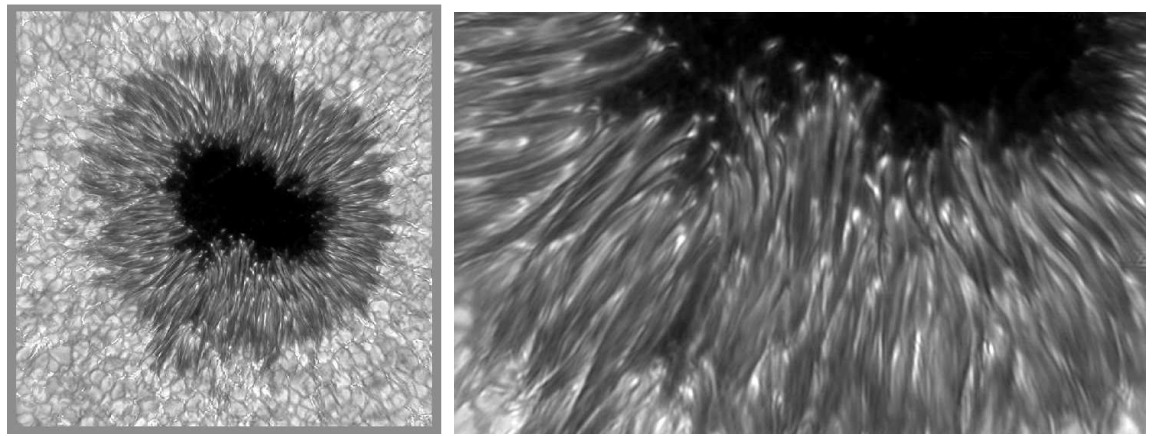

Figure 1. Speckle reconstructed sunspot observed at the DST using the high-order AO (HOAO) system. Courtesy: F. Woeger, T. Rimmele, M. Komsa, C. Berst, S. Fletcher, \& S. Hegwer: NSO/AURA/NSF.

Few years before, resolving structures on the Sun to better than an arcsecond for longer durations were not possible even at good sites due to the Earth's atmosphere. The scenario changed with the advancement of the Adaptive Optics (AO) technology. Hence, modern telescopes thrive on improving the spatial, spectral, and temporal resolutions using an AO system. It may be stated that most of the recent large solar ground based telescope (aperture $>=50 \mathrm{~cm}$ ) designs have an integrated AO system. The realisation of the day-time AO technology lagged behind the night-time due to the difficulties caused by the extended nature of the source as well as the poor seeing conditions prevail during the day-time (Rimmele, 2004; Keller, 2005).

The first solar AO system (Acton, 1992 \& 1993) was developed at Lockheed and tested at the Dunn Solar Telescope (DST). This system worked with high contrast features like solar pores and hence severely limited the scientific use. The development of the correlating Shack-Hartmann wavefront sensor, for the low-order AO system at DST (Rimmele, 2000), has changed the perspective of the solar AO. All solar AO systems currently in operation are based on the correlating Shack-Hartmann sensor. Following Keller (2005), Table I lists the successful AO systems at different solar observatories around the globe. Figure 1 shows an example Sunspot image observed under good seeing condition using a high-order AO (HOAO) system operated at DST. A small portions of the bottom penumbral region is blown-up to show the high spatial resolution achieved with this observation.

\section{Science Achievements}

There are innumerable science observations that made use of the AO. Only few observations are briefly discussed in this paper due to page restriction. The readers are requested to go through the cited references for details. 
Table 1: A list of Successful AO Systems.

\begin{tabular}{|c|c|c|c|c|c|c|c|c|}
\hline $\mathrm{AO}$ & $\begin{array}{l}\text { Act- } \\
\text { uator }\end{array}$ & $\begin{array}{c}\text { Sub- } \\
\text { aperture }\end{array}$ & Camera & $\begin{array}{l}\text { Frame } \\
\text { Rate }\end{array}$ & Hardware & DM & Ref. & $\begin{array}{l}\text { First } \\
\text { Light }\end{array}$ \\
\hline $\begin{array}{c}76 \mathrm{~cm} \\
\text { DST } \\
\text { Lockheed }\end{array}$ & 57 & 19 & - & Analog & - & - & $\begin{array}{l}\text { Acton } \\
1992\end{array}$ & 1986 \\
\hline $\begin{array}{l}76 \mathrm{~cm} \\
\text { DST } \\
\text { LOAO }\end{array}$ & 97 & 24 & - & $<1.6 \mathrm{kHz}$ & $\begin{array}{c}24 \\
\text { DSPs }\end{array}$ & $\begin{array}{c}\text { Xenitics } \\
\text { Inc. }\end{array}$ & $\begin{array}{c}\text { Rimmele } \\
2000\end{array}$ & 1998 \\
\hline $\begin{array}{l}48 \mathrm{~cm} \\
\text { SVST }\end{array}$ & 19 & 19 & $\begin{array}{c}\text { Dalsa } \\
\text { CA-D6 }\end{array}$ & $955 \mathrm{~Hz}$ & $\begin{array}{l}\text { 566MHz } \\
\text { Alpha }\end{array}$ & $\begin{array}{l}\text { AOPTIX } \\
\text { Tech. Inc. }\end{array}$ & $\begin{array}{l}\text { Scharmer } \\
2000\end{array}$ & 1999 \\
\hline $\begin{array}{c}76 \mathrm{~cm} \\
\text { DST } \\
\mathrm{HOAO}\end{array}$ & 97 & 76 & $\begin{array}{l}\text { Custom } \\
\text { built } \\
\text { CMOS }\end{array}$ & $2.5 \mathrm{kHz}$ & $\begin{array}{c}40 \\
\text { DSPs }\end{array}$ & $\begin{array}{c}\text { Xenitics } \\
\text { Inc. }\end{array}$ & $\begin{array}{c}\text { Rimmele } \\
2003\end{array}$ & 2002 \\
\hline $\begin{array}{l}70 \mathrm{~cm} \\
\text { VTT } \\
\text { KAOS }\end{array}$ & 35 & 36 & $\begin{array}{c}\text { Dalsa } \\
\text { CA-D6 }\end{array}$ & $955 \mathrm{~Hz}$ & $\begin{array}{l}\text { 8X900MHz } \\
\text { Sun }\end{array}$ & $\begin{array}{l}\text { Laplacian } \\
\text { Optics }\end{array}$ & $\begin{array}{c}\text { von der Luhe } \\
2003\end{array}$ & 2002 \\
\hline $\begin{array}{c}1.5 \mathrm{~m} \\
\text { McMath } \\
\text { Low Cost }\end{array}$ & 37 & $120-200$ & $\begin{array}{c}\text { Dalsa } \\
\text { CA-D6 }\end{array}$ & $955 \mathrm{~Hz}$ & $\begin{array}{c}1 \mathrm{GHz} \\
\text { Pentium }\end{array}$ & Okotech & $\begin{array}{l}\text { Keller } \\
2003\end{array}$ & 2002 \\
\hline $\begin{array}{l}97 \mathrm{~cm} \\
\mathrm{SST}\end{array}$ & 37 & 37 & $\begin{array}{c}\text { Dalsa } \\
\text { CA-D6 }\end{array}$ & $955 \mathrm{~Hz}$ & $\begin{array}{c}1.45 \mathrm{GHz} \\
\text { Athlon }\end{array}$ & $\begin{array}{l}\text { AOPTIX } \\
\text { Tech. Inc. }\end{array}$ & $\begin{array}{c}\text { Scharmer } \\
2003\end{array}$ & 2003 \\
\hline $\begin{array}{l}65 \mathrm{~cm} \\
\text { BBSO } \\
\text { HOAO }\end{array}$ & 97 & 76 & $\begin{array}{c}\text { Custom } \\
\text { Built } \\
\text { CMOS }\end{array}$ & $2.5 \mathrm{kHz}$ & $\begin{array}{c}40 \\
\text { DSPs }\end{array}$ & $\begin{array}{l}\text { Xenitics } \\
\text { Inc. }\end{array}$ & $\begin{array}{l}\text { Denker } \\
2007\end{array}$ & 2004 \\
\hline
\end{tabular}

\subsection{High Resolution Imaging}

Narrow band G-band images are the first images from most of the AO systems. This is due to the presence of high contrast small-scale features at this wavelength band. However, watching the AO corrected images in the broad-band video camera, attached to the AO system, is always a pleasure for observers. Contrast of these images are usually enhanced using post processing techniques: speckle reconstruction, phase-diversity, or long-exposure point spread deconvolution with or without frame selection. Each of these techniques has its own advantages and disadvantages.

One of the best high spatial resolution observation achieved during the initial phase of the $\mathrm{AO}$ development is the detection of dark cores in the penumbral filaments (Scharmer et al., 2002) using the Swedish Solar Telescope (SST). The observations are obtained using a low order AO system along with real-time frame selection and subsequent image restoration technique. Observations done with HOAO at DST confirmed the existence of dark cored penumbral filaments (see Figure 1). These observations show that the dark cores are unresolved with a cross-section of about $90 \mathrm{~km}$ or smaller and their length can be as long as $1000 \mathrm{~km}$. They can live for atleast an hour. Apart from the dark cores in the penumbral filaments, other thin dark structures, named as 'canals' and 'hairs' are also observed. There are other interesting observations from the SST over the last few years. Few examples are: The 3D structures of small-scale fields (Lites et al., 2004), 

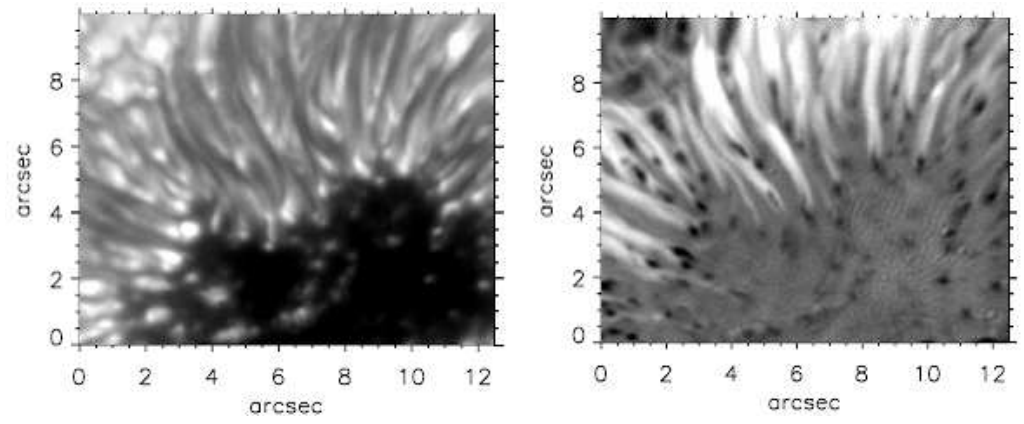

Figure 2. Intensity (right) and velocity (left) image of an observed sunspot penumbral filaments. Dark (bright) in the velocity image represents an upflow (downflow).

leakage of photospheric oscillations and flows to form spicules (de Pontieu, Erdelyi, \& James, 2004), multi-line spectroscopic study of the dark cored filaments (Bellot Rubio, Langhans, \& Schlichenmaier, 2005), and the detailed study of the inclination of magnetic fields and flows in the sunspot penumbrae (Langhans et al., 2005).

High resolution time sequence observation of velocity fields in the penumbral regions (Rimmele \& Marino, 2006a) has allowed one to quantitatively compare different penumbral models. The time sequence clearly show that the upflows (in the footpoints of penumbral dark filaments) and horizontal flow (the Evershed flow) move around and evolve as a unit, indicating that they are part of the same feature. These observations also produce strong evidence that the penumbral grains are the inner footpoints of the Evershed flows where the hot upflow occurs. A full vector magnetic field observations along with such velocity observations may well be able to quantitatively differentiate and refine the few existing models of the penumbra. Such kind of observations can be expected in the near future. Penumbral asymmetries are also studied in detail with observations obtained using AO (Tritschler et al., 2004; Soltau et al., 2005). These recent high spatial resolution penumbral observations have already started the debate on the suitability of the existing penumbral models (Spruit \& Scharmer, 2006; Thomas et al., 2006).

Success of the AO has initiated the deployment of several new backend instruments over the past few years. The Interferometric Bidimensional Spectrometer (IBIS) is one such instrument deployed at the DST in 2003. This instrument produces high spatial, spectral, and temporal resolution observations both in the photosphere and in chromosphere (Cavallini \& Reardon, 2006). Analysing the time sequence observations obtained with this instrument, Vecchio et al. (2007) have concluded that waves with frequencies above the acoustic cut-off propagate to upper layers only in restricted areas of the quiet Sun. Their results support that the network magnetic elements 

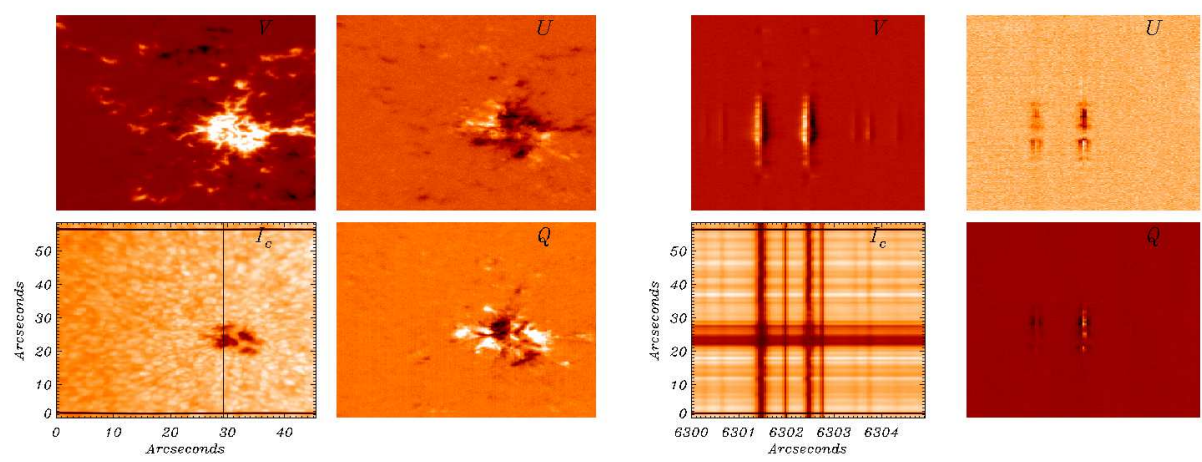

Figure 3. Raster image of the continuum intensity and the Stokes images (left). The maps are produced by averaging a small wavelength region in the continuum for intensity map and in the blue wing of the Stokes profiles for Q, U, and V-maps. The right image shows the Stokes spectrum for the slit represented as a dark vertical line in the continuum intensity image at the left.

can channel low-frequency photospheric oscillations into chromosphere, thus providing a way to input mechanical energy in the upper layers.

\subsection{High Resolution Spectroscopy}

The real test for any solar AO comes from the spectroscopic and spectropolarimetic observations due to the long exposure times and the time required for scanning the region of interest. Moreover, the raster scan images obtained with a spectrograph does not undergo any special processing (like the speckle reconstruction or phase-diversity). In most cases, the solar AO helps in keeping consistent image quality during the scanning time of the spectrograph. The scanning time can be as large as 1-hour depending on the region of interest. The initial observations using the low-order AO corrected images along with the Advanced Stokes Polarimeter (ASP) has revealed small-scale needle-like convective structures around a pore (Sankarasubramanian \& Rimmele, 2003). The radius of the ring like upflows seen around the small-pore matched quiet well with the pattern observed in the magnetohydrodynamic numerical simulations (Steiner, 1998). Figure 3 shows a raster image obtained by scanning the Diffraction Limited Spectro-Polarimeter (DLSP; Sankarasubramanian et al., 2004) operating at the DST along with the HOAO. This raster image is an example for the consistent performance of the HOAO for long (45-minute for this case) duration observations.

Regular observations of diffraction-limited vector magnetic field are feasible using the HOAO and DLSP. These high spatial resolution vector magnetic field measurements are started to produce quantitative informations about small-scale magnetic elements, like umbral dots (Socas-Navarro, 2004; 

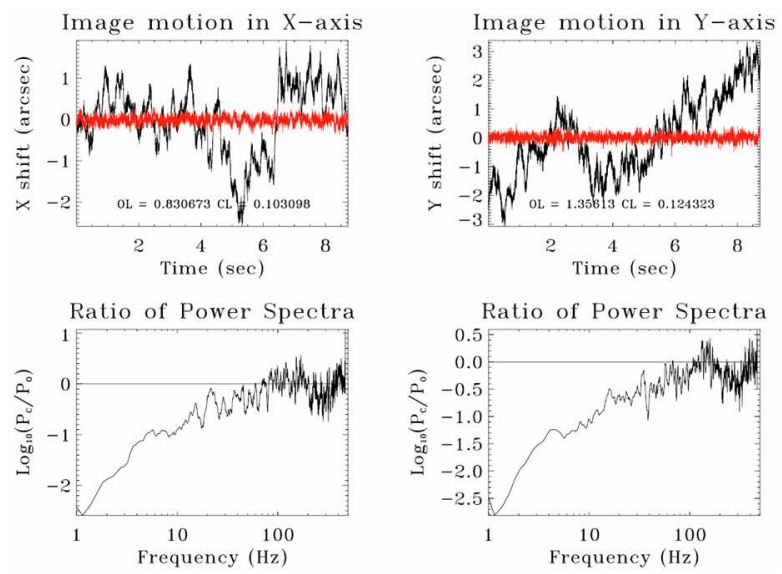

Figure 4. A closed-loop tip-tilt correction of the USO-AO system. A closed-loop update rate of $1 \mathrm{kHz}$ is used. Achieved closed-loop bandwidth is about $100 \mathrm{~Hz}$ and the improvement in the 'rms' image motion is anywhere between 5 to 25 .

Sankarasubramanian, Rimmele, \& Lites, 2004), small-scale magnetic elements in and around active regions (Sankarasubramanian \& Hagenaar, 2007) and quiet sun magnetic fields (Lites \& Socas-Navarro, 2004). There are other similar spectroscopic instruments developed at the AO corrected image planes, examples are the POLIS and TIP (Schmidt et al., 2003; Martinez Pillet et al., 1999). The development of the AO has also helped in obtaining very high resolution spectroscopic observations in the near and far infra-red regions (Keller et al., 2003 \& Lin, private communication).

\section{AO Developments in India}

The development of AO technology was started very recently in India. A low-cost AO system along with a Shack-Hartmann wavefront analyser was developed and tested in the laboratory (Ganesan et al., 2005). A $10 \times 10$ sub-apertures for sampling the wavefront and a 37-channel MMDM from OKO Tech was used in this system. This system cannot be used for solar observations due to the slow read-out speed of the camera (25 fps).

Udaipur Solar Observatory (USO) is involved in developing an Adaptive optics system for their $50 \mathrm{~cm}$ aperture Multi-Application Solar Telescope (MAST; Venkatakrishnan 2007). A laboratory setup has been developed for testing the whole system. A $15 \mathrm{~cm}$ telescope is used as a light feed for the laboratory optical set-up. A portion of the image from this $15 \mathrm{~cm}$ telescope is selected, by using a field stop, for imaging on to a science as well as a wavefront sensing camera after going through a tip-tilt mirror and a plane mirror 
(later this plane mirror will be replaced with a deformable mirror). The wave-front sensing camera can operate at a rate of $1 \mathrm{kHz}$. The algorithm for estimating the image motion is implemented using MMX instructions and a PID type control system is developed for the tip-tilt mirror control. The system was tested in the laboratory using a laser source as well as using the solar image. Closed loop tip-tilt correction was achieved and Figure 4 shows the performance of the system during a closed-loop operation. Experiments are going on to include the deformable mirror and a closed loop AO correction with the DM is expected to happen in the middle of 2007.

\section{Future Needs}

The overwhelming scientific observations using the AO effected several solar observatories (IRSOL, THEMIS, GREGOR, NST, \& USO) to develop an AO system for their respective telescopes and they are expected to be functional over the next few years. The two newly proposed large telescope NLST (this proceedings) and ATST (Wagner et al., 2006) will require a system with an order more compared to the one currently operating. ATST has proposed for an AO system with 1280 sub-apertures and 1369 actuators and expected to be operated in a closed-loop bandwidth of $250 \mathrm{~Hz}$ (Rimmele et al., 2006b).

One of the major limitation of the currently operating AO systems is the inability to achieve diffraction limited imaging over a larger field-of-view (FOV). The image becomes blurred away from the isoplanatic patch and the size of this patch (usually about 10") can vary from site to site and also from day to day. The difficulty in consistently locking on to the low-contrast features like granulation is the second major disadvantage with the current AO systems. The faster evolution of the granules compared to high-contrast structures poses this difficulty. The performance of the AO reduces when the observations are done close to the limb and the current $\mathrm{AO}$ cannot be used for coronal observations.

Multi-conjugate Adaptive Optics (MCAO) has been proposed as a technique to increase the FOV over which the corrections are done. The Sun is an ideal object to carry out the MCAO due to its extended nature. Solar MCAO efforts are currently underway at the NSO and at Kipenheuer Institute of Sonnenphysick (KIS). There are considerable progress made during the last two years in realising the MCAO (Rimmele et al., 2006c; Berkefeld, Soltau, \& von der Luhe, 2006).

\section{Acknowledgements}

The author KS acknowledges the following personnel for providing the necessary informations: Balasubramaniam, K. S. (NSO), Bayanna, R. (USO), Schlichenmaier, R. \& Sigwarth, M. (KIS). 


\section{References}

Acton, D. S., \& Dunn, R. B., 1993, SPIE, 1920, 348.

Acton, D. S., \& Smithson, R., 1992, Appl. Opt., 31, 3161.

Bellot Rubio, L., Langhans, K., \& Schlichenmaier, R., 2005, Astron. Astrophys., 443, L7.

Berkefeld, T., Soltau, D., \& von der Luhe, O., 2006, SPIE, 6272, 4.

Cavallini, F., \& Reardon, K., 2006, Mem. S.A.It. Suppl., 9, 55.

Denker, C. et al., 2007, PASP, 119, 170.

de Pontieu, B., Erdelyi, R., \& James, S., 2004, Nature, 430, 536.

DeRosa, M., 2005, In Sankarasubramanian et al., editors, Large-scale Structures and their Role in Solar Activity, page 337, ASP, San Francisco.

Ganesan, A. et al., 2005, Journal of Optics, 34, 67.

Keller, C., 2005, In Brandner \& Kasper, editors, Science with Adaptive Optics, page 119, Springer, Berlin.

Keller, C., Plymate, C., \& Ammons, S. M., 2003, SPIE, 4853, 351.

Langhans, K. et al., 2005, Astron. Astrophys., 436, 1087.

Lites, B. et al., 2004, Solar Phys., 221, 65.

Lites, B., \& Socas-Navarro, H., 2004, Astrophys. J., 613, 600.

Martinez Pillet, V. et al., 1999, In Rimmele, et al., editors, High Res. Solar Physics:

Theory, Observations, and Techniques, page 264, ASP, San Francisco.

Rimmele, T., \& Marino, J., 2006a, Astrophys. J., 646, 593.

Rimmele, T. et al., 2006b, SPIE, 6272, 33.

Rimmele, T. et al., 2006c, SPIE, 6272, 5.

Rimmele, T., 2004, SPIE, 5490, 34.

Rimmele, T. et al., 2003, SPIE, 4839, 635.

Rimmele, T., 2000, SPIE, 4007, 218.

Sankarasubramanian, K., \& Hagenaar, H., 2007, BASI, submitted.

Sankarasubramanian, K. et al., 2004, SPIE, 5171, 207.

Sankarasubramanian, K., Rimmele, T., \& Lites, B., 2004, AAS, 204, 2006.

Sankarasubramanian, K., \& Rimmele, T., 2003, Astrophys. J., 598, 689.

Scharmer, G. et al., 2003, SPIE, 4853, 370.

Scharmer, G. et al., 2002, Nature, 420, 151.

Scharmer, G. et al., 2000, SPIE, 4007, 239.

Schmidt, W. et al., 2003, AN, 324, 300.

Schussler, M., \& Vogler, A., 2006, Astrophys. J., 641, L73.

Socas-Navarro, H. et al., 2004, Astrophys. J., 614, 448.

Soltau, D. et al., 2005, In Brandner \& Kasper, editors, Science with Adaptive Optics, page 129, Springer, Berlin.

Spruit, H., \& Scharmer, G., 2006, Astron. Astrophys., 447, 343.

Stein, R., \& Nordlund, A., 2006, Astrophys. J., 642, 1246.

Steiner, O. et al., 1998, Astrophys. J., 495, 468.

Thomas, J. et al., 2006, Astron. Astrophys., 452, 1089.

Tritschler, A et al., 2004, Astron. Astrophys., 415, 717.

von der Luhe, O. et al., 2003, SPIE, 4853, 187.

Vecchio, A. et al., 2007, Astron. Astrophys., 461, L1.

Venkatakrishnan, P., 2007, BASI, Submitted.

Wagner, J. et al., 2006, SPIE, 6267, 9. 